\title{
Tratamiento térmico del acero inoxidable ferrítico AISI 430L sinterizado en atmósfera de $\mathrm{N}_{2}-\mathrm{H}_{2}$
}

\author{
J.M. Ruiz-Román", F. Corpas", F.J. Iglesias", L.E.G. Cambronero* y J.M. Ruiz-Prieto*
}

\begin{abstract}
Resumen Los aceros inoxidables ferríticos, son aceros que contienen esencialmente cromo (12-30\%) junto con níquel y molibdeno en cantidades que, en general, no sobrepasan el $1 \%$ y, en ocasiones, se les adicionan otros elementos como son el aluminio, silicio, titanio o niobio. En el presente trabajo de investigación se ha evaluado la influencia del nitrógeno presente en la atmósfera de sinterización sobre la microestructura y propiedades del acero inoxidable ferrítico AISI 430L. Además, se ha realizado un tratamiento térmico posterior de hipertemple y maduración con el objeto de incrementar las propiedades mecánicas y a corrosión de este acero inoxidable, mediante la modificación microestructural de los nitruros complejos de hierro y cromo precipitados durante la etapa de sinterización.
\end{abstract}

Palabras clave Metalurgia de polvos. Acero inoxidable. Tratamiento térmico. Nitrógeno.

\section{Heat treatment of AISI 430L stainless steel sintered in $\mathrm{N}_{2}-\mathrm{H}_{2}$ atmosphere}

\begin{abstract}
In this Work the influence of sintering AISI 430L stainless steel in $\mathrm{N}_{2}-\mathrm{H}_{2}$ atmosphere on its properties and microstructure have been studied. The presence of nitrogen in the sintering atmosphere leads to complex nitrides formation the AISI 430L sintered steel microstructure. A subsequent heat treatment was applied to modify this microstructure in order to improve the mechanical properties and corrosion resistance of these ferritic stainless steels.
\end{abstract}

Keywords Powder metallurgy. Stainless steel. Heat treatment. Nitrogen.

\section{INTRODUCCIÓN}

Los aceros inoxidables sinterizados, debido a su elevada porosidad interconectada o aislada, presentan una mayor superficie libre que los productos convencionales, por lo que son susceptibles de reaccionar con la atmósfera del horno de sinterización dando lugar a compuestos que producen una disminución de sus propiedades químicas finales ${ }^{[1 \mathrm{y}}{ }^{2]}$.

En el caso concreto de este trabajo, el nitrógeno presente en la atmósfera de sinterización difunde rápidamente a las elevadas temperaturas que se encuentran los compactos, por lo que estos se encuentran superficialmente saturados de este elemento y, si la velocidad de enfriamiento no es lo suficientemente rápida, se producirá la precipitación $^{[3-5]}$ de nitruros de cromo en los granos de ferrita, con la consiguiente disminución de cromo en la matriz y, por tanto, un empeoramiento de la resistencia a corrosión.

Además, la presencia de estos nitruros complejos de forma laminar origina la formación de zonas con diferente potencial de oxidación ${ }^{[6]}$ con lo que la resistencia a corrosión también se ve mermada.

Debe recordarse que la solubilidad del nitrógeno presente en la atmósfera de sinterización ${ }^{[7]}$, en aleaciones de hierro, depende, básicamente, de la composición y estructura cristalina de la aleación y de la temperatura y presión del horno durante el proceso de sinterización. En lo que respecta a la composición de la aleación, la solubilidad del nitrógeno aumenta con el contenido en manganeso, cromo y molibdeno de la misma, disminuyendo con el contenido en níquel, carbono y silicio.

En el presente trabajo de investigación se pretende desarrollar un tratamiento térmico que

(*) Universidad Politécnica de Madrid. Departamento de Ingeniería de Materiales. Rios Rosas 21, 28003 Madrid.

$\left(^{* *}\right)$ E.U. Politécnica de Linares. Dpto. de Ingeniería Química, Ambiental y de los Materiales. Alfonso X el Sabio 28, 23700 Linares (Jaén).

Rev. Metal. Madrid Vol. Extr. (2005) 269-273 
permita modificar la estructura cristalina y la cantidad de estos precipitados obtenidos durante la etapa de sinterización, de forma que se consiga aprovechar la parte positiva de la absorción de nitrógeno como es un incremento de las propiedades mecánicas ${ }^{[8]}$ sin perjudicar la resistencia a corrosión de este tipo de $\operatorname{aceros}^{[9 \text { y } 10]}$.

\section{MATERIALES Y PROCEDIMIENTO EXPERIMEN- TAL}

La materia prima empleada fue polvo de acero inoxidable ferrítico AISI 430L, de la casa HÖGANÄS de la siguiente composición: $16,9 \% \mathrm{Cr} ; 0,1 \% \mathrm{Ni}$; $0,18 \% \mathrm{Mn} ; 1,16 \%$ Si y $0,018 \%$ C.

La compactación de los polvos se realizó de forma uniaxal en matriz flotante, con lubricación, mediante estearato de cinc, en las paredes de la misma y controlando la velocidad de aplicación de la carga. La compactación se realizó a una presión de $700 \mathrm{MPa}$, obteniéndose probetas normalizadas de tracción MPIF Standard 10 (ASTM E-69) y probetas cilíndricas normalizadas MPIF Standard 45 (ASTM B331) para los ensayos de corrosión.

A continuación, las probetas fueron sinterizadas en horno tubular a $1.250^{\circ} \mathrm{C}$ durante $30 \mathrm{~min}$ a una velocidad de $5{ }^{\circ} \mathrm{C} / \mathrm{min}$ y atmósfera de $95 \%$ $\mathrm{N}_{2}-5 \% \mathrm{H}_{2}$.

Finalmente, y con el objeto de modificar la morfología eutectoide de los nitruros complejos de hierro y cromo precipitados durante la etapa de sinterización se procede a la realización de un tratamiento de solubilización, hipertemple y maduración, consistente en un calentamiento a $1.150^{\circ} \mathrm{C}$ en atmósfera de argón, mantenimiento de la temperatura $30 \mathrm{~min}$ y enfriamiento posterior en agua. Como temperaturas de maduración se seleccionaron desde $100{ }^{\circ} \mathrm{C}$ hasta $700{ }^{\circ} \mathrm{C}$ en intervalos de $100^{\circ} \mathrm{C}$, en atmósfera inerte de argón.

En cuanto a las propiedades evaluadas fueron las siguientes:
- Propiedades físicas del acero inoxidable ferrítico AISI 430L (densidad, porosidad total ${ }^{[11]}$, Porosidad superficial y variación dimensional en estado sinterizado.

- Propiedades mecánicas estáticas (dureza, resistencia a tracción, límite elástico y alargamiento) en todos los estados descritos anteriormente de sinterización, temple y maduración de la aleación AISI 430L.

- Resistencia a corrosión mediante ensayos de inmersión ( $\mathrm{HCl} 35 \%, \mathrm{H}_{2} \mathrm{SO}_{4} 50 \%, \mathrm{NaCl} 5 \%$ ) sin aireación y a temperatura ambiente, excepto el de cámara salina (ASTM B-117-73).

Debido a que el procesado normal de ese tipo de aceros, por lo explicado con anterioridad, se realiza sinterizando en atmósfera de vacío, se fabricaron muestras sinterizadas en horno de vacío a las que se evaluaron las mismas propiedades, con el objeto de tener en todo momento una referencia comparativa de los resultados obtenidos y poder comprobar la viabilidad del método seleccionado.

\section{RESULTADOS Y DISCUSIÓN}

En la tabla I se recogen los resultados de las propiedades físicas y mecánicas evaluadas del acero inoxidable ferrítico AISI 430L sinterizado en atmósfera de $95 \% \mathrm{~N}_{2}-5 \% \mathrm{H}_{2}$ y de vacío.

De estos primeros resultados, destacar que se confirma lo establecido anteriormente. Al sinterizar en atmósfera de nitrógeno, la presencia de los nitruros complejos de hierro y cromo provoca un aumento en las propiedades mecánicas frente al mismo sinterizado en vacío, junto con una sensible disminución del alargamiento a rotura y de la variación dimensional del acero sinterizado. Esta presencia de nitruros complejos de forma laminar la podemos comprobar en la micrografía (Fig. 1a), donde se encuentran, a lo largo de toda la microestructura, precipitados en los granos de ferrita.

Tabla I. Propiedades físicas y mecánicas del acero inoxidable ferrítico AISI $430 \mathrm{~L}$ sinterizado a $1.250^{\circ} \mathrm{C}$ en atmósfera de vacío

$$
\text { y } \mathrm{N}_{2}-\mathrm{H}_{2}
$$

Table I. Physical and mechanical properties of AISI $430 \mathrm{~L}$ stainless steel sintered in vacuum and $\mathrm{N}_{2}-\mathrm{H}_{2}$ atmosphere to $1250^{\circ} \mathrm{C}$

\begin{tabular}{lccccccc}
\hline $\begin{array}{c}\text { Atmósf. } \\
\text { De Sint. }\end{array}$ & $\begin{array}{c}\text { Densidad } \\
\left(\mathbf{g} / \mathbf{c m}^{3}\right)\end{array}$ & $\begin{array}{c}\text { Porosidad } \\
\text { total (\%) }\end{array}$ & $\begin{array}{c}\text { Porosidad } \\
\text { superficial }\end{array}$ & $\begin{array}{c}\text { Variación } \\
\text { dimensional }\end{array}$ & Rt (MPa) & $\begin{array}{c}\text { Alargamiento } \\
(\%)\end{array}$ & HV \\
\hline Vacío & 6,80 & 11,6 & 7,3 & $-2,9$ & 445 & 10,3 & 103 \\
$\mathrm{~N}_{2}-\mathrm{H}_{2}$ & 6,76 & 12,2 & 9,8 & $-1,4$ & 508 & 1,0 & 242 \\
\hline
\end{tabular}


Tratamiento térmico del acero inoxidable ferrítico AISI $430 \mathrm{~L}$ sinterizado en atmósfera de $\mathrm{N}_{2}-\mathrm{H}_{2}$ J.M. Ruiz-ROMÁN, F. CORPAS, F.J. Iglesias, L.E.G. CAMBRONERO y J.M. Ruiz PRIETO
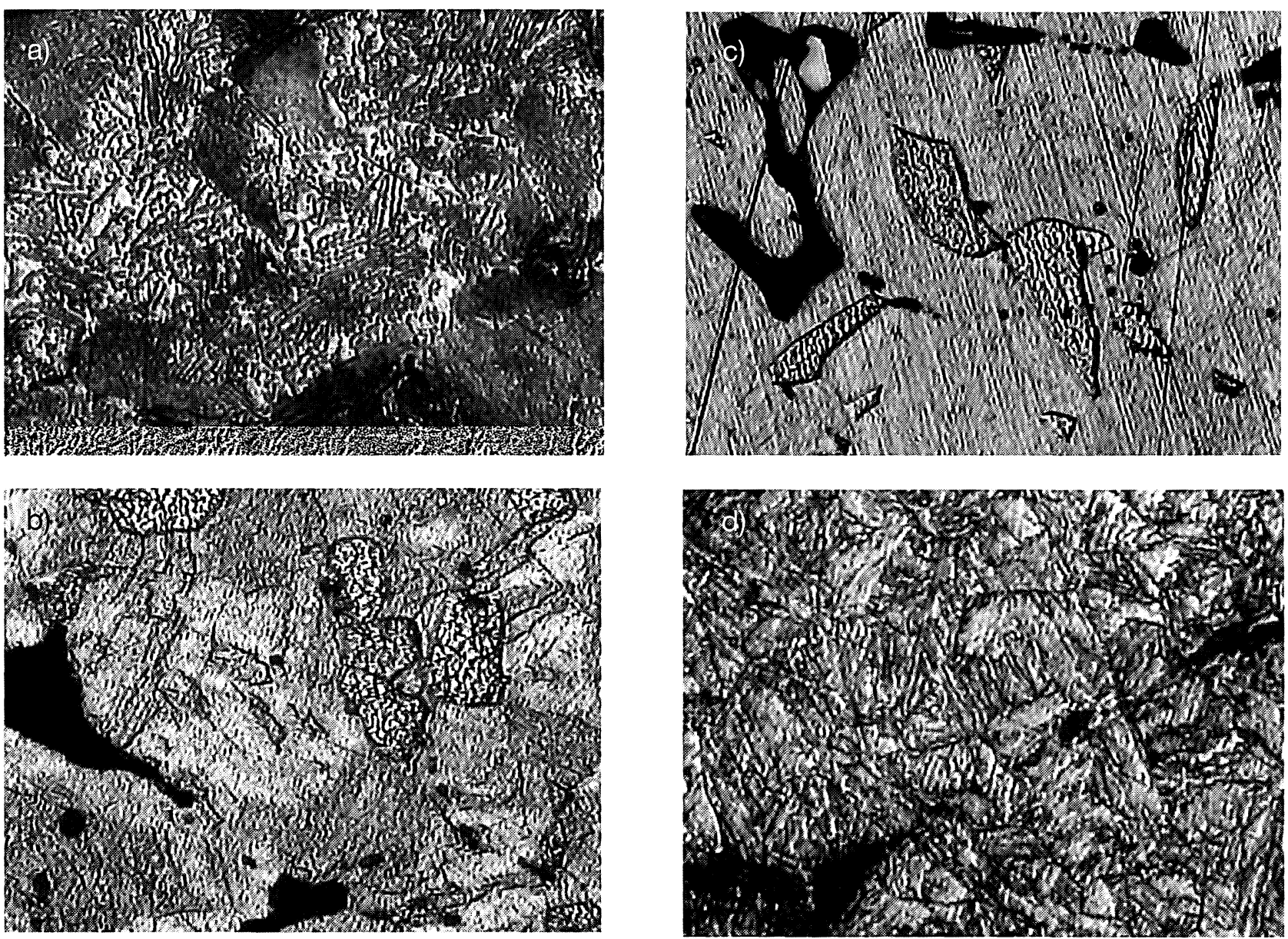

Figura 1. Microestructura del acero inoxidable ferrítico AISI 430L, a) sinterizado en atmósfera de $\mathrm{N}_{2}-\mathrm{H}_{2}$, b) templado, c) madurado a $300^{\circ} \mathrm{C}$ y d) madurado a $700^{\circ} \mathrm{C}$.

Figure 1. Microstructure of AISI $430 \mathrm{~L}$ stainless steel, a) sintered in $\mathrm{N}_{2}-\mathrm{H}_{2}$, b) tempered, c) heating to $300{ }^{\circ} \mathrm{C}$ and d) heating to $700^{\circ} \mathrm{C}$

A continuación, en la tabla II se recogen los resultados de los ensayos de corrosión medidos como peso perdido por unidad de área y día, realizados sobre el acero AISI 430L en su estado sinterizado tanto en vacío como en nitrógeno-hidrógeno.

Tabla II. Comportamiento a corrosión frente a distintos medios del acero inoxidable ferrítico AISI $430 \mathrm{~L}$ sinterizado en vacío y en atmósfera de $90 \% \mathrm{~N}_{2}-10 \% \mathrm{H}_{2}$

Table II. Corrosion resistance in different corrosive means of AISI $430 \mathrm{~L}$ stainless steel sintered in vacuum and $\mathrm{N}_{2}-\mathrm{H}_{2}$ atmosphere of $90 \%$

\begin{tabular}{|c|c|c|c|c|}
\hline $\begin{array}{l}\text { AISI } \\
430 \mathrm{~L}\end{array}$ & $\begin{array}{c}\text { ASTM } \\
\text { B-117-73 }\end{array}$ & $\begin{array}{c}\text { Agua de mar } \\
\qquad \begin{array}{c}\mathrm{NaCl} 5 \% \\
\mathrm{~g} / \mathrm{m}^{2}\end{array}\end{array}$ & $\begin{array}{c}\mathrm{HCl} 35 \% \\
\text { díag } / \mathrm{m}^{2} \cdot \text { día }\end{array}$ & $\begin{array}{c}\mathrm{H} 2 \mathrm{SO} 450 \% \\
\mathrm{~g} / \mathrm{m}^{2} \cdot \text { día }\end{array}$ \\
\hline Vacío & 110 & 2,33 & 725 & 1270 \\
\hline $\mathrm{N}_{2}-\mathrm{H}_{2}$ & 201,04 & 3,4 & 2327,89 & 14,31 \\
\hline
\end{tabular}

Como era de esperar, el nitrógeno presente en la atmósfera de sinterización presenta un efecto muy perjudicial sobre la resistencia a corrosión del acero inoxidable AISI 430L. Hay que indicar que en los ensayos de corrosión por picaduras se evaluó la velocidad de corrosión como la pérdida en peso por unidad de tiempo y área ya que el ataque que se presentó fue generalizado y no se pudo determinar ni el tamaño ni la densidad de picaduras presentes.

A continuación, se procede a tratar térmicamente las muestras sinterizadas en nitrógeno-hidrógeno mediante solubilización, hipertemple y maduración posterior, obteniéndose los resultados que se recogen en las tablas III y IV. La micrografía de la figura 1b) muestra cómo los nitruros complejos laminares quedan disueltos en la ferrita mediante este tratamiento. Del mismo modo, en la micrografía de la figura 1c) podemos ver cómo junto a los granos de ferrita aparecen precipitados 
Tratamiento térmico del acero inoxidable ferrítico AISI 430L sinterizado en atmósfera de $\mathrm{N}_{2}-\mathrm{H}_{2}$ J.M. RuIz-ROMÁN, F. CORPAS, F.J. IgLESIAS, L.E.G. CAMBRONERO y J.M. RUIZ PRIETO

Tabla III. Propiedades mecánicas del acero inoxidable AISI $430 \mathrm{~L}$ sinterizado en atmósfera de $\mathrm{N}_{2}-\mathrm{H}_{2}$ a $1.250^{\circ} \mathrm{C}$ templado y madurado a distintas temperaturas de tratamiento

Table III. Mechanical properties of AISI $430 \mathrm{~L}$ stainless steel sintered in $\mathrm{N}_{2}-\mathrm{H}_{2}$ atmosphere to $1250^{\circ} \mathrm{C}$ and heat treatment to differents temperature

\begin{tabular}{lcccc}
\hline Estado & $\begin{array}{c}\text { Dureza } \\
\text { HRB }\end{array}$ & $\begin{array}{c}\text { Limite } \\
\text { elástico } \\
\text { MPa }\end{array}$ & $\begin{array}{c}\text { Resistencia } \\
\text { a la tracción } \\
\text { MPa }\end{array}$ & $\begin{array}{c}\text { Alarga- } \\
\text { miento } \\
\%\end{array}$ \\
\hline Templado & 63 & 171 & 400 & 0,9 \\
Madur. $100^{\circ} \mathrm{C}$ & 66 & 176 & 401 & 0,9 \\
Madur. $200^{\circ} \mathrm{C}$ & 66 & 194 & 454 & 0,6 \\
Madur. $300^{\circ} \mathrm{C}$ & 66 & 175 & 478 & 0,8 \\
Madur. $400^{\circ} \mathrm{C}$ & 68 & 168 & 496 & 1,0 \\
Madur. $500^{\circ} \mathrm{C}$ & 69 & 193 & 518 & 1,0 \\
Madur. $600^{\circ} \mathrm{C}$ & 59 & 202 & 602 & 0,9 \\
Madur. $700^{\circ} \mathrm{C}$ & 52 & 193 & 480 & 0,8 \\
\hline
\end{tabular}

Tabla IV. Resistencia a corrosión del acero inoxidable AISI $430 \mathrm{~L}$ sinterizado en atmósfera de $\mathrm{N}_{2}-\mathrm{H}_{2}$ a $1.250^{\circ} \mathrm{C}$ templado y madurado a distintas temperaturas de tratamiento

Table IV. Corrosion resistance of AISI $430 \mathrm{~L}$ stainless steel sintered in $\mathrm{N}_{2}-\mathrm{H}_{2}$ atmosphere to $1250^{\circ} \mathrm{C}$ and heat treatment to differents temperature

\begin{tabular}{|c|c|c|c|c|}
\hline AISI 430L & $\begin{array}{c}\text { ASTM } \\
\text { B-117-73 }\end{array}$ & $\begin{array}{l}\text { Agua de mar } \\
\qquad \mathrm{NaCl} 5 \%\end{array}$ & $\begin{array}{l}\mathrm{HCl} 35 \% \\
\mathrm{~g} / \mathrm{m}^{2} \cdot \text { día }\end{array}$ & $\begin{array}{c}\mathrm{H}_{2} \mathrm{SO}_{4} 50 \% \\
\mathrm{~g} / \mathrm{m}^{2} \cdot \text { día }\end{array}$ \\
\hline Temple & 140 & 11 & 1866 & 90 \\
\hline Madur. $100^{\circ} \mathrm{C}$ & 136 & 12 & 2103 & 17 \\
\hline Madur. $200^{\circ} \mathrm{C}$ & 134 & 14 & 1873 & 11 \\
\hline Madur. $300^{\circ} \mathrm{C}$ & 130 & 8 & 121 & 5 \\
\hline Madur. $400^{\circ} \mathrm{C}$ & 160 & 12 & 3264 & 22 \\
\hline Madur. $500^{\circ} \mathrm{C}$ & 124 & 20 & 1927 & 15 \\
\hline Madur. $600^{\circ} \mathrm{C}$ & 100 & 2 & 2138 & 14 \\
\hline Madur. $700^{\circ} \mathrm{C}$ & 175 & 4 & 1659 & 86 \\
\hline
\end{tabular}

nitruros complejos pero en proporción muy inferior al material sinterizado, mientras que a $700{ }^{\circ} \mathrm{C}$ esos nitruros vuelven a tomar forma laminar y se encuentran totalmente precipitados igual que en estado sinterizado.

En lo que hace referencia a los resultados en las propiedades mecánicas, la tendencia lógica es un incremento en la resistencia mecánica relacionada con la temperatura de maduración, debido a la mayor presencia de fase precipitada efectiva en cuan- to al mecanismo de endurecimiento por precipitación se refiere. Cuando la maduración se realiza a $700{ }^{\circ} \mathrm{C}$, la microestructura resultante corresponde a la misma del acero simplemente sinterizado, los precipitados dejan de ser coherentes cristalográficamente con la matriz, lo que se corresponde con unas resistencias mecánicas similares. Respecto a los resultados del ensayo de dureza, se puede hacer una reflexión similar aunque es un dato que en estos materiales sinterizados puede no presentar un comportamiento tan lineal como es la resistencia mecánica, debido a la porosidad que presentan.

Como se comentó con anterioridad, la presencia de los nitruros complejos de hierro y cromo precipitados en el acero inoxidable ferrítico AISI 430L provocan una disminución de la resistencia a corrosión respecto a la que tiene este mismo acero sinterizado en vacío y, por tanto, sin ningún contenido en nitrógeno. Este fenómeno se acentúa cuanto mayor es la cantidad en fase precipitada presente. Sin embargo, se produce un fenómeno muy interesante al producirse un aumento sensible a la resistencia a corrosión del acero inoxidable $430 \mathrm{~L}$ templado y madurado a $300{ }^{\circ} \mathrm{C}$ respecto a la resistencia que presenta este mismo acero sinterizado en vacío, en especial frente a los medios clorhídrico y sulfúrico. Se consigue, pues, a esta temperatura de maduración, un equilibrio entre la fase precipitada y el nitrógeno aun disuelto en el acero, que permite un incremento de la resistencia a la corrosión del mismo frente a los medios anteriormente citados.

\section{CONCLUSIONES}

Se pueden extraer las siguientes conclusiones:

- El nitrógeno presente en la atmósfera de sinterización, se absorbe en los aceros inoxidables ferríticos AISI 430L durante este proceso, precipitándose durante el enfriamiento en forma de nitruros complejos de hierro y cromo laminares.

- El tratamiento térmico de solubilización temple y maduración a $300^{\circ} \mathrm{C}$ permite obtener aceros inoxidables sinterizados AISI 430L con resistencia mecánica superior a los sinterizados en atmósfera de vacío.

- Del mismo modo, el tratamiento térmico de solubilización, temple y maduración a $300{ }^{\circ} \mathrm{C}$ permite obtener aceros inoxidables sinterizados AISI 430L con resistencias a corrosión superiores en medio clorhídrico y sulfúrico a los sinterizados en vacío. 
Tratamiento térmico del acero inoxidable ferrítico AISI 430L sinterizado en atmósfera de $\mathrm{N}_{2}-\mathrm{H}_{2}$ J.M. Ruiz-ROMÁN, F. CORPAS, F.J. IgLESIAS, L.E.G. CAMBRONERO y J.M. RUIZ PRIETO

\section{Agradecimientos}

Los autores quieren agradecer a la Universidad Politécnica de Madrid por su apoyo económico a este trabajo de investigación.

\section{REFERENCIAS}

[1] J.A. Bas, C. Molins y J. Planas, Adv. Powder Metall. 5 (1992) 385-397.

[2] J.M. Ruiz PRIETO, Powder Metall. 37 (1994) 57-60.

[3] J. Rawers, J. Bennet y R. DOAN, Acta Metall. 40 (1992) 1.195-1.199.

[4] A. Lopez, A. Alvarez De Sotomayor, E.J. Herrera, Rev. Metal. Madrid 37 (2001) 315-325.
[5] H. Wriedt y N. GoKCen, Bull. Alloys Phase Diagram 8 (1987) 355.

[6] A. Bautista, F. Velasco, Proc. PM2TEC-2002, Vol. 7, Orlando, EEUU, MPIF, 2002, pp. 134-142.

[7] F. Biancaniello, Proc. PM2TEC-2002, Vol. 3, Orlando, EEUU, MPIF, 2002, pp. 198-206.

[8] M. Rosso, Proc. PM2TEC-2002, Vol. 7, Orlando, EEUU, MPIF, 2002, pp. 102-109.

[9] F. Velasco, J.M. Ruiz-Roman, Brit. Corros. J. 31295 299.

[10] F. Velasco, J.R. Ibars, J.M. Ruiz-Roman, Corrosion 52 (1996) 47-52.

[11] M. Martínez, C.M. Abreu, M.J. Cristóbal, P. Merino, X.R. NovoA, G. Pena y M.C. Pérez. Rev. Metal. Madrid 38 (2002) 315-325. 